\title{
AMENDMENTS
}

\section{Author Correction: Novel Hexb-based tools for studying microglia in the CNS}

Takahiro Masuda (1), Lukas Amann, Roman Sankowski ${ }^{(1 D}$, Ori Staszewski, Maximilian Lenz, Paolo d’Errico,

Nicolas Snaidero, Marta Joana Costa Jordão (1), Chotima Böttcher (10), Katrin Kierdorf, Steffen Jung (1), Josef Priller,

Thomas Misgeld, Andreas Vlachos, Melanie Meyer-Luehmann (1D, Klaus-Peter Knobeloch (D) and Marco Prinz

Correction to: Nature Immunology https://doi.org/10.1038/s41590-020-0707-4, published online 15 June 2020.

In the version of this article initially published, the hyphen was missing from author Melanie Meyer-Luehmann's surname. The error has been corrected in the HTML and PDF versions of the article.

Published online: 11 August 2020

https://doi.org/10.1038/s41590-020-0774-6

(c) The Author(s), under exclusive licence to Springer Nature America, Inc. 2020

\section{Author Correction: ORF8 and ORF3b antibodies are accurate serological markers of early and late SARS-CoV-2 infection}

Asmaa Hachim, Niloufar Kavian (D), Carolyn A. Cohen, Alex W. H. Chin, Daniel K. W. Chu, Chris K. P. Mok, Owen T. Y. Tsang, Yiu Cheong Yeung, Ranawaka A. P. M. Perera, Leo L. M. Poon, J. S. Malik Peiris and Sophie A. Valkenburg (i)

Correction to: Nature Immunology https://doi.org/10.1038/s41590-020-0773-7, published online 17 August 2020.

In the version of this article initially published online, in the section "Lack of cross-reactivity of existing human coronavirus responses for N, ORF3b and ORF8 by LIPS," the $\beta$-human coronavirus (HCoV) OC43 was incorrectly identified as an $\alpha$-HCoV and the $\alpha$-HCoVs 229E and NL63 were incorrectly identified as $\beta$-HCoVs. In the Methods section "Patients and samples collection," it was incorrectly stated that plasma samples were collected from 84 patients with COVID-19. A total of 84 plasma samples were collected from 61 patients with COVID-19. The errors have been corrected in the print, PDF and HTML versions of the article.

Published online: 27 August 2020

https://doi.org/10.1038/s41590-020-0788-0

(c) The Author(s), under exclusive licence to Springer Nature America, Inc. 2020 